\title{
Erratum to: 50 Years of Fluxoid Quantization: 2e or Not $2 \mathrm{e}$
}

\section{Dietrich Einzel}

Published online: 18 May 2011

(C) Springer Science+Business Media, LLC 2011

Erratum to: J Low Temp Phys (2011) 163: 215-237 DOI 10.1007/s10909-011-0349-x

There is an error in the equation that appears at the bottom of page 220 in the original article. The corrected equation is as follows:

$$
n^{\mathrm{s}}(T)=n[1-Y(T)] ; \quad Y(T)=\frac{1}{2 k_{\mathrm{B}} T} \int_{0}^{\infty} \frac{d \xi_{\mathbf{p}}}{\cosh ^{2}\left(\sqrt{\xi_{\mathbf{p}}^{2}+\Delta^{2}(T)} / 2 k_{\mathrm{B}} T\right)}
$$

The online version of the original article can be found under doi:10.1007/s10909-011-0349-x.

D. Einzel ( $\square)$

Walther-Meissner-Institut, Bayerische Akademie der Wissenschaften, 85748 Garching, Germany e-mail: Dietrich.Einzel@wmi.badw-muenchen.de 